\title{
TE MATARUA O TE HANGARAU
}

\author{
Dean P. S. Mabuta* \\ Rachael Ka'ai-Mabuta ${ }^{\dagger}$
}

\section{He whakarāpopoto}

Kua hau ngā rongo o te hangarau i te mata o te whenua, me uaua ka kitea he tangata kāore i te whakamahi i tētahi momo hangarau—te pouaka whakaata, te waea pūkoro, te rorohiko, me te ipurangi. Katoa ēnei mea he uri nō te wāhiaotanga, ā, he whakangāwari i te horapa o ngā mea katoa ki ngā hau e whā o te ao. Ko te ito o tēnei tuhinga roa he titiro ki ngā mata e rua o te hangarau, arā, te mata i tāmi i te iwi Māori, me te mata i whakawhanake i te iwi Māori. Ko ngā whakahau ka whakatakotoria e māua i tuhia hei whakaohooho, hei whakatenatena i te iwi Māori kia kaua e waiho mā te ao te tuakiri me te reo Māori e waihanga ki te ao.

\section{He kupu matua}

hangarau, matihiko, te reo Māori, tuakiri Māori, mātauranga Māori

\section{Ngā pātaritari a te hangarau matihiko}

Kua whakatauritehia te wāhiaotanga ki te tāmitanga i runga anō i āna whakaaweawenga kino kua pā ki ngā hapori, me ngā iwi taketake o te ao. Ko te hangarau matihiko tētahi tohu, tētahi taputapu nui o te wāhiaotanga, ā, kua kaha whakararu ia i ngā wāhanga o te ahurea Māori. E kitea ana i tēnei āhuatanga te whakatuanui haere o te reo kua horapa whānui mā te ipurangi; ngā raru kua puta i te apohanga ahurea me te taiōritehanga; tae atu ki te rite tonu o te whakaparori i ngā tāngata taketake mā te hē o ngā kōrero, arā, kāore i te motuhenga.

* Ngāti Mahuta, Waikato. Senior Lecturer, Te Ipukarea-National Māori Language Institute, Auckland University of Technology (AUT), Auckland, New Zealand imēra:dmahuta@aut.ac.nz

† Ngāti Porou, Ngāi Tahu, Native Hawaiian, Cook Island Māori. Senior Lecturer, Te Ipukarea—National Māori Language Institute, Auckland University of Technology (AUT), Auckland, New Zealand

DOI: 10.20507/MAlJournal.2017.6.3.9 
Nā te horapa o te ipurangi me te wāhiaotanga i wātea ai te ahurea Māori ki te marea ahakoa te iti, te kore rānei i mōhio ki tōna hītori. Ko tā te iwi Māori whakahaere, tiaki i te rere o ngā kōrero mō āna tikanga me tōna ahurea, kāore i te tino kaha. Inā te hiranga o te aronui a te Māori ki te whakarite kaupapa e hoki mai ai te mana motuhake o ōna mātauranga Māori kei runga i te ipurangi.

Heoi anō rā, koinei te wāhiaotanga me tana pātanga ki runga i te iwi Māori, otirā, ngā iwi taketake o te ao. Ko ngā taonga a Tāne-rore me Te Rēhia anō hoki tētahi āhuatanga kua rongo i te ngau o te wāhiaotanga. Kei whenua kē a Tāne-rore e haka ana, à, e tukua ana a Te Rēhia kia hurahura ki mua i kāhui tauiwi o whenua kē. Tērā te kōrero a Brown (2007), kia takatū ake te iwi Māori, kei riro mā tikanga kē à tàtou taonga, '. . . the technologies are applied to them, and their culture, by another group' (wh. 78).

Ko ēnei kupu a Brown e hāngai ana ki te whakaaturanga o te āhua, o te tuakiri Māori ki runga i te ipurangi, tae atu ki ngā momo hangarau pēnei i ngā īpae. Ko te kaupapa o āna kōrero, me mātua aro te iwi Māori ki ēnei hangarau kei riro mā iwi kē ā tātou tikanga, tō tātou reo, tō tātou tuakiri e hangahanga, e whakairi hoki hei tirohanga mā te ao whānui. He momo āhuatanga tēnei e whakaatu ana i tā te wāhiaotanga mahi ki ngā mātauranga Māori, arā, nā te māmā o te whakamahi i te ipurangi me te tere hoki o te rere o te mātauranga ki whenua kē, ki rohe kē, i tino whai pānga ai ki te hanganga o te tuakiri o te tangata.

Kia tirohia ake te tauira o te ipae ataata, o Tiriata, he ippae kua hau ngā rongo i te ao Māori, otirā i te ao hurihuri. Ka tuhia ana te kupu haka ki te pouaka rapu, ka ohorere te tangata $\mathrm{i}$ te mahi a te ataata e whakaatu ana i te haka. Ka mutu, ehara i te mea he Māori e haka ana, kāo, ko te nuinga o ngā ataata haka e kitea ana, he ope ākonga nō ngā kura tuarua o Amerika e 'haka' ana i tētahi haka whiu ringa, hāmama, hāparangi, aha atu rānei. Ka puku te rae, ka kārangirangi i tēnei āhuatanga nā te mea, ko te nuinga o ngā ākonga kei ngā ataata nei nō Te Moana-nui-a-Kiwa, ā, he Māori te āhua, he reo moutere hoki e puta ana i te waha. Ko te whakaaro, he ākonga Hāmoa ēnei kua tipu i raro i te maru whakahaere o Amerika, ā, he hiahia nō rātou ki te whakaihiihi i a rātou anō i mua i ā rātou kēmu whutupōro, ka haka. Tuatahi, e kite atu ana a iwi kē i ēnei momo haka me te mīharo atu ki te haka a te Māori. Tuarua, kua waihangatia mai, kua whakapaetia mai e te hunga mātakitaki te āhua, te tuakiri Māori i roto i ō rātou hinengaro. Heoi anō rā, ko te mate nui, kāore te Māori tūturu i whakaatu i te haka nei, engari nā kūware, nā hākawa, nā iwi kē!

I te mutunga ake, ko te mana o te ipurangi, o ngā hangarau katoa tēnei e whakaata nei i ngā haka a te Māori ki whenua kē. Ka kitea te haka a te Māori i runga i ngā ìpae, me te rongonui o ngā momo haka nei, kāore e taea e te iwi Māori te karo, te kaupare rānei ngā mahi a te hunga hiahia i ēnei taonga. Nā konā te whakahau o runga ake nei, kia māia, kia mataara a Ngāi Māori ki te whakamahi i te hangarau, kia kaua rawa e waiho mā kūware mā e whakatauira atu te haka a ō tātou tīpuna Māori ki te ao, mā iwi kē rānei te tuakiri Māori e waihanga hei kai mā te ao whānui.

Mehemea ko te wāhiaotanga te taniwha, ko te pakihitanga ōna niho, arā, te pakihitanga o tō tātou ahurea. Ki te whakahāngaitia ki ngā kōrero o tēnei wāhanga, ko te whakamahi i ngā haka, i ngā waiata a te Māori hei taputapu mahi moni mā Tauiwi. I roto i Aotearoa nei, i whenua kē hoki, kua rite tonu te pēneitia, arā, te whakamahia o ngā waiata a te Māori. Hei tauira, ka tahuri ki a Air New Zealand, arā, i ngā tau ngahuru 90, i whakamahia e rātou te waiata 'Pōkarekare ana', hei waiata motuhake mō tā rātou kamupene huri, huri i te ao, mea rawa ake ka whakamanahia hei tohu tuakiri mō te kamupene (Ka'ai-Mahuta 2010). Nā te rongonui o tēnei waiata i whiwhi pūtea ai a Air New Zealand, me tō rātou mōhio, nā te Māori anō i rongonui ai te waiata nei. I kaha waiatatia e ngā pēne Māori o mua, e Kiri Te Kanawa anō 
hoki i a ia e oriori haere ana i te ao, ka mutu, kua mōhiotia te waiata nei ki ngā tōpito o te ao. Nō reira, e whiwhi moni ana a Tauiwi i ngā mahi a te Māori.

Nā tēnei momo pakihitanga i whakataputapuhia ai te mātauranga Māori. Ko te tikanga o tēnei āhuatanga, he tukanga whakaemiemi mātauranga kia whakataputapuhia hei mea hokohoko. Tērā tētahi mātanga Kanaka Māoli, a Haunani-Kay Trask, nāna te kōrero, ko tēnei momo tukanga, ko te 'kairautanga o te ahurea' ('prostitution of culture') (Trask 1999, wh. 137). I te nuinga o te wā ko ngā mahi whakataputapu i te reo me ngā tikanga Māori tētahi mahi kāore i whakahaerengia e te Māori. Whakatūpato ana a Tā Tīpene O'Regan (2001) i a ia e kōrero ana mō tōna ake iwi, mō Ngāi Tahu:

I believe that Ngai Tahu heritage and history is part of our rangatiratanga, and that our runanga are the guardians of that ... I am concerned that iwi must find ways to bring the intellectual and cultural property of Maori under some greater cultural control (wh. 35).

Kua roa ngā rangatira, pēnei i a Tā Tīpene, e kōrero ana i ō rātou āwangawanga mō ngā mana whakairo hinengaro, mana ahurea Māori, tae atu hoki ki te whakahorapatanga o ngā tikanga me te hītori Māori (O'Regan 2001, wh. 36-37). I te kaha tūkinotia o à tātou taonga e ngā kairangahau Pākehā, e whakapono ana ètahi Māori me kaua rawa e tuku ō tātou kōrero tuku iho kia tuhia, kia kapohia, kia horapa rānei ki te ipurangi, māna, kia mātua mau i te Māori te mana motuhake o ērā tukanga.

E whia kē nei ngā tau a Māori mā e whakapau kaha ana kia ora anō te ahurea Māori, pēnei i te reo. Ko te hiahia, kia whakaakona te reo ki te tini me te mano kia pakari anō ai te reo $\mathrm{i}$ waenga i te iwi Māori, ā, kāore i tino tutuki. Ka mutu, kia hoki anō ki tēnei mea te hangarau, te taniwha i whakatuanui ai i ō tātou wāhi Māori ki te reo Pākehā. Nā, kia tōaitia te whakahau, te pūtake o tēnei wāhanga o te tuhinga nei, arā, me tahuri tātou, te iwi Māori, ki te whakamahi i te hangarau hei rākau whakamāori i a ia anō. Ko te hiahia kia whakatuanui i èrā paepae ipurangi, i ērā paepae matihiko ko te reo Pākehā te matua, e reo Māori ai rātou, ā, kia tuku mā te ringa Māori tūturu e tārai te tuakiri Māori ka kitea whānuitia huri noa i tēnei ao hurihuri. Koinei te uho o tēnei tuhinga, à, ka whakawhānui haeretia, ka whakapūmautia hoki ēnei kōrero i ngā wāhanga e whai ake nei.

\section{Kia urutau ki te huringa o te ao}

Ahakoa te kōrero a ètahi mātanga, e kī ana he iwi mārō te iwi Māori, kāore ōna hiahia kia huri tōna āhua, kia rerekē, i whakaatu ngā tīpuna Māori i ō rātou āheinga. Nā ngā tīpuna Māori tonu te urutau ki ngā āhuatanga hou i whakatauira. Nō mai anō tēnei momo, nō te wà o ngā tohunga hōpara, nā rātou i whakaatu mai 'an ongoing process of adaptation and cultural development in new and changing locations' (Howe 2003, wh. 70). Tārake ana te kitea o tēnei āhuatanga i te urutaunga a te Māori ki ngā taiao makariri, me ngā taiao taikaha o Aotearoa nei ka tau mai ana rātou ki ngā moutere mahana o Te Moana-nui-a-Kiwa.

Kua kitea i roto i te heketanga o Hìtori, he tere a Māori ki te whakamahi i ngā hangarau me ngā pūkenga hou mehemea e kite ana rātou he pāinga ōna ki te whanaketanga o tō rātou iwi. Ko tētahi tauira nui o tēnei āhuatanga ko tā te Māori kapo atu i te pūkenga tuhituhi i te wā o ngā kura mihinare. I tere whakamahi ō tātou tīpuna Māori o taua wā rā i ō rātou pūkenga hou hei oranga mō ngā uri whakaheke. I te rautau tekau mā iwa, he rite tonu tā te Māori tuhituhi, ka whakahāngai ai i ngā pūkenga ā-waha ki te perehi, ā, i kaha tuhituhi i ngā whakapapa, i ngā hītori ā-iwi, i ngā kōrero tikanga, me ngā waiata. Waihoki, tāpae atu ana ki ngā tuhinga tawhito, inā te nui o ngā niupepa Māori, arā, neke atu i te 40 ngā niupepa reo Māori motuhake i tāngia mai i te tau 1840 tae noa mai ki te rautau rua tekau. I roto i ēnei 
niupepa ko ngā tuhinga e pā ana ki ngā take tōrangapū, ngā take pāpori, ngā reta ki te ètita, ngā poroporoākī me ngā tangi apakura anō hoki (McRae 2004, wh. 137). I te mutunga iho, i tahuri hoki ngā tīpuna Māori ki te whakamahi i ngā edison wax cylinders hei taputapu kapo i te reo waiata.

\section{Tā ngā iwi taketake whakamahi i te hangarau matihiko}

Whakatata mai ana ki ēnei tau nei, kua tahuri ngā iwi taketake ki te whakamahi i te hangarau matihiko i te mea he taputapu pai hei whakahorapa kōrero, ā, hei taonga tūhono, tuitui hoki i ngā tāngatao te hapori. Nā konā i kīia ai he taonga hirahira mō ngā reo me ngā ahurea taketake. E hāngai pū ana tēnei nā te kaha peinga me ngā tainukunuku o te nuinga o ngā tāngata taketake, ka hua ana ko ngā hapori tūāporoporo, pēnei i te iwi Māori e noho ana ki Ahitereira. E whakapae ana ngā tatauranga, kotahi i roto i te ono tāngata Māori e noho ana ki Ahitereira i tēnei wā.

Ko tētahi āhuatanga nui o te ao Māori, ānō nei he mātāpono, ko tēnei mea, te whanaungatanga, arā, ko ngā herenga whakapapa i waenga i tēnā tangata, i tēnā tangata, i tēnā tangata. Nā, ko te pūtake o ngā īpae, kia makere iho ngā rohenga whenua, kia pai ai tā te tangata hono atu ki te ao. Nō reira, koirā te kaupapa o tēnei wāhanga, he matapaki i tā te hangarau matihiko tuitui, whatu i te korowai tangata.

Waihoki, ehara i te mea ko tā te hangarau he tuitui noa iho i ngā whanaungatanga o te iwi Māori e noho tūāporoporo nei i te ao mō te tūhonohono noa iho te take, kāo. Engari he titiro ki ngā hua o ngā īpae hei kukume mai i te reo Māori, otirā ngā reo mōrearea katoa o te ao, i te pari o te rua. Me whakatakoto i konei ètahi tauira e whakaatu ana i te āhei o ngā ìpae ki te whakakotahi i te iwi Māori, i te iwi kōrero Māori anō hoki kia whanake ai.

Kua takoto te kōrero, ko te aronga nui o te kāhui whakarauora reo i ngā tau o mua, ko te whakaako o te reo Māori ki te tangata kia mātinitini anō ai te iwi kōrero Māori. Heoi anō, ko tētahi raru nui i hua ake i tēnei kāhui i kitea i te wā ka puta ana ngā tauira i te akomanga, ko te nuinga kāore i whai hoa kōrero Māori, ka waiho mā Tāwhiri-matangi-rau rātou e pupuhi ki ngā hau e whā. Nā konā i toko ake te pātai, ka rere marara ana ngā tauira kōrero Māori, me pēhea te whakapūmau tonu i èrā taura here tangata?

$\bar{A}$ kāti, koinei māua e toro atu nei ki ngā īpae, arā, ngā paepae o te hangarau matihiko o tēnei whakatupuranga, kia mana ai te kōrero, 'ko te rākau whakatuanui i te reo Māori, koia hoki te rākau e pakari anō ai te reo Māori’.

Ko tētahi mahi nui a te hangarau matihiko, ko te whakatūtū haere i ngā hapori ā-ipurangi, nā te mea, kāore te nuinga o tātou i te noho ki rō papa kāinga, ki rō puni. Mō te taha ki ngā rautaki whakarauora reo, arā, ērā e whakamahi ana i ngā ìpae, kua tipu ake ēnei hapori kōrero Māori ki runga ipurangi. Kāore i te aukatia ā rātou 'whakawhitiwhiti' (ā-kupu nei) e ngā rohe tauārai papa whenua, ā, e whakatauira ana i te whakamahinga o tētahi taputapu tāmi reo Māori hei taputapu whakaora, whakawhanake i ngā rautaki whakarauora reo. Nā konā i tahuri atu ai ki te kaupapa ipae e whai ake nei.

\section{Ngā îPae me ngā hapori ipurangi reo Māori}

Nā Fishman te kōrero, ka ora te reo i ngā whakatupuranga ki te whakapūmautia ki roto i te kāinga, ka mutu, he ariā kua roa rawa e tautokotia ana e ngā kōkō tatakī, e ngā mumu reo katoa o ngā tau e whā tekau kua taha ake nei. Waihoki, ko ngā hapori inamata o te reo Māori, arā, ngā marae, ngā papa kāinga, kua kī pohapoha i te reo Pākehā nā ngā hangarau matihiko o te wā. Ko ērā whānau e mārō ana ki te whakarumaki i ō rātou whare ki te reo Māori, e whawhai ana me ènei hangarau matihiko kua tomo atu ki te kāinga. Nā, e whakaaweawe pū ana tēnei āhuatanga $\mathrm{i}$ te uara, $\mathrm{i}$ te mana o te 
reo Māori o ia rā, o ia rā. Koinei te tino mana o ngā hangarau matihiko, ahakoa te pai, te nui rānei o te reo Māori o tō whare, mehemea he hangarau kei reira, kāore e taea e koe te reo Pākehā te karo.

Nā konā te hapori ngākaunui ki te reo Māori i tīmata ai ki te whakamahi i ngā īpae ki te kōrero Māori, ki te whakamāori, arā, ka ekea atu ēnei wāhi ko te reo Pākehā te reo matua. Mà tēnei te mana o te reo Māori e whakapakari.

I roto i ngā tau tata kua araara mai ètahi hapori īpae ki te kawe i te reo Māori ki ngā tōpito katoa o te ipurangi, à, ko te matua o ènei paepae ipurangi ko Pukamata. Heoi anō, e kitea ana hoki te reo Māori ki runga o Pae Tihau, o Atapaki, o Pae Āhua, o Tiriata. Koinei ngā rangatira o te ao o nāianei, o te ao o āpōpō anō hoki, nō reira, ko te painga atu mehemea ka ora te reo i ènei īpae ā haere ake nei.

Āpiti atu ki tēnei, ko ngā rōpū īpae pakari, ko èrā rōpū i whānau mai ai i te hirikapo, i te moemoeā, i te manawanui o ngā tāngata e kōrero Māori ana. Ehara i te mea he rautaki, he kaupapa, he mahere rānei i waihangatia e te kāwanatanga, kāo, i tupu kē i ngā hapori kōingo ki te reo Māori. Nō reira, me pēhea kē hoki e kore ai e matomato te tupu o ēnei iwi matihiko ina tupu mai i te whatumanawa o te tangata! Ko ngā kōhanga reo te tino tauira o tēnei whakaaro, he mea tupu tēnei kaupapa rangatira i te ngākau o te iwi, kāore i tatari kia tahuri mai te kāwanatanga-nā ngā koroua, ngā kuia, ngā mātua ngā kōhanga reo tuatahi i whakarite. I whakamahia e rātou te taputapu e kïia nei ko te mātauranga-te rākau kua roa e patu ana i te iwi Māori, i te reo Māorihei rākau whakaora i te reo me te iwi Māori whānui.

Ki te hoki anō ki te whare o te rangahau, me āna tikanga, ka whakahuatia ngā kupu 'virtual intercultural bridgework', me te 'virtual cosmopolitanism' (Sobre-Denton, 2015). Ko ēnei momo kupu te waha whakamārama i ngā tūhonohono tangata kua tupu mai i ènei īpae hou, arā, he hono i ārahina e te taputapu matihiko. Hei tā Gajjala (2012), kua kaha ake tā ngā iwi taketake raweke i te ipurangi me ngā īpae huri noa i te ao. Ko te ngākau Māori ka whakaae ki tēnei whakapae a Gajjala, i te mea, inā te nui o te mana o te whakapapa, o te whanaungatanga o tō tātou ao Māori, ka ngāwari tā te Māori toro atu ki tētahi taonga, ki tētahi hangarau ko tōna pūtake he whiri i ngā taura here i waenga i ngā tāngata. Ka puta te kōrero i a Sorrell (2013), mō te pai o ngā hangarau matihiko; '. . . to facilitate intercultural connections among friends and intimate partners to meet, develop friendships, and maintain contact particularly at great geographical distances' (wh. 166).

Ko te tino raruraru e pā nei ki te reo Māori, ko te noho tūāporoporo a te iwi kōrero Māori. I te nuinga o te wā e rāwaho ana te nuinga o te iwi Māori, heoi anō rā, ahakoa tēnei toimahatanga ko te ngākau Māori e kimi tonu ana i êrā taura here e tau ai ia i tēnei ao, a kāti, ko te reo Māori anō hoki tērā. Ko te tino koha o te hangarau ki te reo Māori ko te tuku i ana taura kia toro atu i te ao ka herea, ka whiria ki ngā reo tūāporoporo kia kotahi mai anō ki raro i te tāhuhu kotahi.

Ka mutu, e haere tahi ana ēnei mahi katoa a ngā īpae, arā, te whakaora i te reo me te whakapūmau i ngā taura here tangata mā te whakarite rōpū, whakarite whārangi Pukamata. Waihoki, katoa ngā hìtori pāpori o ēnei rā kei runga i te ipurangi. Ko ètahi atu wāhi e kitea ana ngā huinga tāngata matihiko ko ngā paetukutuku à-iwi, ngā rauemi matihiko whakaako reo, ngā rangitaki wānanga/tōrangapū, ngā hautaka ipurangi, ā, katoa ēnei āhuatanga e whakakaha ana i te whakahorapatanga o te mātauranga ki ngā iwi taketake whānui tonu. Nō reira kia tahuri ake ināianei ki ētehi kōrero mō tā te tangata tiaki i ō tātou mātauranga matihiko.

\section{Te tiaki i ngā kohinga matihiko o ngā reo taketake}

Pupuke ake nei te whakakapinga o te tai kikokiko ki te tai matihiko. Ko tētahi wāhanga nui o tā tātou waihanga kōrero e kawea ana i 
runga ipurangi. He tere ake, he ngāwari ake, $\bar{a}$, he iti ake te utu ki te whāngai mātauranga mā ngā hangarau matihiko, ka mutu, i ēnei rā ko ngā whare matihiko anake te wāhi e kitea ai ètahi o ō tātou mātauranga. Heoi anō, koia hoki te take he taonga paraheahea ngā taonga matihiko, he ngāwari rawa te panoni, te whakakore, te whakangaro hoki, i runga anō i te mōhio, i ètahi wā ko taua kape matihiko rā te kape motuhake!

I te nuinga o te wā, raru ai ngā kaupapa ipurangi taketake i te korenga o rātou e whiwhi pūtea tautoko. Ka kati ana ērā kaupapa ipurangi, ka ngaro ngā mātauranga i noho i runga i èrā paepae ipurangi, ka motu rānei te here, me te āhei o te iwi ki te kuhu atu ki ngā pātaka kōrero rā. Nā reira, pūrangiaho ana me mātua tiaki ngā mātauranga ka tukuna à-matihiko ki te iwi kia pūmautia mō ake tonu atu, ā, mā ngā uri e haere ake nei.

Mō te iwi Māori, ko te oranga o te ahurea Māori kei roto i te tiakanga o ōna mātauranga, $\bar{a}$, e tika ana me mātua whakarite te tangata i tēnei rangi tonu mō ngā hiahia o āpōpō. Koinei te tūāpapa o têtahi o ā māua kaupapa e kīia ana ko Tāmata Toiere, he paetukutuku, he pātaka kōrero kua whakakīkīngia ki ngā waiata me ngā haka kua rangahaua, kua whakaemihia, kua tiakina hei tuku ki te iwi whānui.

He tino taputapu te ipurangi hei whakamahi mā te iwi Māori, me te maumahara hoki ki te mau tonu te Māori ki tōna mana i te paepae nei, mā te Māori anō te mātauranga Māori e whakairi ki te ipurangi, ki ngā īpae rānei o te ao. Mā te iwi Māori anō te tuakiri o te Māori e whakatauira ki te ao, à, koinei tētahi o ngā tino mātāpono o Tāmata Toiere. Mā Tāmata Toiere e whakatauira atu ki te ao me pēhea te rangahau tika i ngā mātauranga Māori, arā, he wāhi e taea ai e te tangata te toro atu ki ngā whakamahuki mō tētahi waiata, ngā kupu tika, tae atu hoki ki te waiatatanga tika o tērā waiata, o tērā haka rānei. E ai ki te tohunga rotarota, ki a Albert Wendt, 'We are what we remember; society is what it remembers, that's why we must control what we remember-history-and hand that on to our children' (Wendt cited in Sarti 1998, wh. 209).

He mea nui mō tātou, mō te iwi taketake, kia mōhio tātou me pēhea te tiaki i ō tātou kōrero mā te hangarau matihiko. Hei tauira, nā te horapa haere o ngā ìpae-tae rawa atu ki tana hono i te iwi tūāporoporo ki te 'kāinga'ko te nuinga o tō te tangata hìtori pāpori kei te kapohia ki runga ipurangi. Nō reira, me mātua tiaki ēnei hītori pāpori hei taonga mā ngā whakatipuranga o āpōpō.

He mahi nui tā ngā ohu ā-motu pēnei i ngā Whare Pukapuka hei ohu tiaki āhuatanga matihiko. Ko ō tātou whare pukapuka ngā pātaka mātauranga o te iwi tangata whānui, ā, ahakoa kua rerekē te waka kawe i tērā mātauranga ki te marea, e mau tonu ana te hiranga o te tiaki i taua mātauranga rā. Mō te iwi Māori tonu e noho ana ki Aotearoa nei, he mahi nui tā ngā whare pukapuka ki te tiaki i te reo o te tangata whenua. He rautaki hoki tēnei mahi ki te whakatairanga i te kanorau o te motu, i te ngākau mahaki o te motu, ā, kia taumarutia, kia whakamanahia hoki ngā tirohanga a iwi kē.

Tērā anō tētahi kōrero a Albert Wendt, 'History has everything to do with memory and remembering: history is the remembered tightrope that stretches across the abyss of all that we have forgotten' (Wendt 1987, wh. 79). Ko ngā mahara e kaha whakapūmautia ana, i te nuinga o te wā, i toko ake i te hapori matua, $\bar{a}$, ki Aotearoa nei, ko te nuinga o èrā mahara he mea whānau mai i te ao Pākehā. Nō reira, kia tōaitia te kōrero i konei, he mea waiwai te tiaki i ngā reo taketake mā te hangarau matihiko. Ka mutu, ko tētahi wāhanga nui o tēnei āhuatanga ko te whakauru i ngā tāngata taketake ki roto pū i ngā tukanga whakahaere e whakamahia ana hei kohikohi, tiaki, whakahorapa i ō rātou ake mātauranga.

\section{Hei Whakakopani}

Nā Tā Apirana Ngata te kōrero: E tipu, e rea, mō ngā rā o tôu ao; 
Ko tō ringa ki ngā rākau a te Pākehā hei ara mō te tinana;

Ko tō ngākau ki ngā taonga a ō tīpuna Māori hei tikitiki mō tō māhunga,

ā ko tō wairua ki tō Atua nāna nei ngā mea katoa.

Ko ngā kupu, 'E tipu e rea', te uho o te whanaketanga o te ao Māori. Takoto ana te kōrero i a Ngata, e pakari ai te Māori mō ngā piki me ngā heke o tōna whakatipuranga, me mau pūmau ki ngā taonga i heke mai i ōna tīpuna Māori, ā, me toro atu ki ērā whanaketanga a te iwi Pākehā hei oranga mōna. Waihoki, he whakahau tēnei nā Ngata kia tahuri te Māori ki ngā hangarau a te Pākehā, engari me Māori tonu te kawe i a ia anō i tēnei ao hurihuri. Ko ngā hangarau matihiko tētahi o ngā whanaketanga e āhei ai te kaupare atu i ngā pātaritari o tēnei whakatipuranga.

Tērā tētahi hui iwi taketake i tū ki Poipiripi, ko 'Puliima National Indigenous Language and Technology Forum' te ingoa, ā, i tae atu māua ki tēnei hui. Ko te kaupapa o tērā hui, ko 'Modern Ways for Ancient Words'. E whakapono ana māua, koinei pea tētahi whakamāramatanga mō ngā kupu a Tā Apirana, arā, he kōrero hei whakaohooho, hei whakatenatena i te iwi Māori ki te kimi i ngā huarahi, e kīia ana, 'modern ways for ancient words'.

Waihoki, ko te tino aronga o ngā kaupapa kua whakatakotoria i tēnei tuhinga, he whakaoho i te whatumanawa o te iwi Māori kia kawea tōna ahurea, otirā tōna reo anō hoki ki ērā wāhi ko te reo Pākehā te matua. Ka mutu, kia tomo atu ki a Tahora-nui-a-Hiko, te rohe whānui e kaha whakapau wā ana ā tātou tamariki. E pēnei ana te kōrero nā te mea kua roa rawa te hunga e ngākaunui ana ki te oranga tonutanga o te reo Māori e whai ana i te whakahau, ka ora te reo i te kāinga, i ngā arero o ā tātou tamariki. Ko tā māua e kī ake nei, anei te whare o ā tātou tamariki, he whare matihiko, nō reira, whāia kia Māori tērā whare.

Āpiti atu ki tēnā, me waiho mā ēnei momo whakaaro e whakaaweawe atu, e whakahōhā atu ngā mahi a te hunga tauiwi kua tere kapo i à tātou tikanga, i à tātou haka, i ā tātou waiata. Mà tātou anō, mā te iwi Māori whānui anō ēnei āhuatanga e whakarite, arā, mā te Māori anō tōna ao e kōrero, e waihanga, e whakahorapa ki te aroaro o te ao hurihuri. Ki te tukua a Tānerore hei taurekareka noa mā Tauiwi, ka toremi ia, otirā tō tātou ao Māori, ki te korokoro o te hangarau. Ki te pukuriri te Māori, ki te amowheke te Māori i ngā ataata, i ngā pikitia ka iri ki te ipurangi, nō tātou, nō te Māori tonu te hē. Kapohia ake ngā rākau a te Pākehā hei oranga mō te tinana, ko ngā taonga tuku iho a kui mā, a koro mā ka inumia hei whakaora i te wairua.

Ka whawhai tonu ngā iwi taketake ki ngā whakapātaritari kua hua mai i te wāhiaotanga me te hangarau matihiko, heoi anō tā tātou he āta takahi i ngā ara o èrā pātaritari rā kia pūmautia ko ngā reo taketake, kia tiakina ko ngā mātauranga taketake, hei aha, hei oranga mō tātou katoa. Takatū ake, Māori mā, takatū ake. 


\section{Rārangi kupu}

apohanga ahurea

Atapaki
ipae
kairautanga
Pae Āhua
Pae Tihau
pakihitanga
taiōritehanga
tāmitanga
Tiriata
wāhiaotanga

Whakataputapu cultural appropriation

nō te kupu 'apo', mehe tangata pukurua e kapo ana i te kai i te tēpu

Snapchat

social media platforms koinei te kupu e kawea whānuitia ana e te hunga kōrero Māori mō ngā paepae pāpāho ā-pāpori

prostitution

Instagram

Twitter

commercialisation

homogenisation

colonisation

YouTube

globalisation

Commodification nō te kupu kairau, āra, he tangata kua huri ki te onioni tangata ki te mahi moni

nō te kupu 'pakihi', arā, ngā kamupene mahi moni ānō nei nō te 'tai' kotahi tātou katoa, arā, he ōrite ngā āhuatanga katoa, kāore ōna motuhaketanga

nō te kupu wāhiao - kua wāhia mai te ao ki te tangata, kua ngāwari te rangawhāwhā atu ki ngā āhuatanga katoa o te ao whānui 


\section{Rārangi pukapuka}

Brown, D. (2007). Theorizing Digital Cultural Heritage: A Critical Discourse. In F. Cameron and S. Kenderdine (Eds). Cambridge: MIT Press.

Gajjala R (2012). Cyberculture and the Subaltern: Weavings of the Virtual and Real. Plymouth, UK: Lexington Books.

Howe, K. R. (2003). The Quest for Origins-Who First Discovered and Settled New Zealand and the Pacific Islands. Auckland: Penguin Books (NZ) Ltd.

Ka'ai-Mahuta, R. T. A. (2010). 'He kupu tuku iho mō tēnei reanga: A critical analysis of waiata and haka as commentaries and archives of Māori political history'. Unpublished PhD. Auckland University of Technology, Auckland.

McRae, J. (2004). Ngā tuhituhinga reo MāoriLiterature in Māori. In T. M. Ka'ai, J. C. Moorfield, M. P. J. Reilly \& S. Mosley (Eds.), Ki te Whaiao: An Introduction to Māori Culture and Society, (pp. 133-138). Auckland: Pearson Education.

O’Regan, T. (2001). Old Myths and New Politics: Some Contemporary Uses of Traditional History.
In J. Binney (Ed.), The Shaping of HistoryEssays from the New Zealand Journal of History, (pp. 15-37). Wellington: Bridget Williams Books Limited.

Sarti, A. (1998). Spiritcarvers: Interviews with eighteen writers from New Zealand. Coss/Cultural 31-Readings in the Post/Colonial Literatures in English series. (Series Editiors: G. Collier, H. Maes-Jelinek \& G. Davis). Amsterdam: Rodopi. Sobre-Denton, M. (2015). 'Virtual intercultural bridgework: Social media, virtual cosmopolitanism, and activist community-building,' In New Media \& Society 1-17. DOI: 10.1177/1461444814567988

Trask, H. (1999). From a Native DaughterColonialism and Sovereingty in Hawai'i. (Revised edition). Honolulu: University of Hawai'i Press. Wendt, A. (1987). Novelists and Historians and the Art of Remembering. In A. Hooper, S. Britton, R. Crocombe, J. Huntsman \& C. Macpherson (Eds.), Class and Culture in the South Pacific, (pp. 78-91). Auckland: Centre for Pacific Studies, University of Auckland and Institute of Pacific Studies, University of the South Pacific. 